\title{
Aspectos da Vida Sexual de Estudantes Adolescentes
}

Sexual Aspects of Adolescent Students

Aspectos de La Vida Sexual de Estudiantes Adolescentes

Sheyla de Amorim Cordeiro da Silva ${ }^{1}$

Jairo Calado Cavalcante ${ }^{2}$

Leila Araújo Barbosa Costa ${ }^{3}$

\section{Resumo}

Objetivo: Descrever aspectos da vida sexual de estudantes adolescentes. Método: Estudo descritivo, transversal, quantitativo, com amostra aleatória de 556 estudantes, ambos os sexos, dos 10 aos 18 anos, oriundos de três escolas públicas de Maceió/AL. Os dados foram coletados através de um questionário semiestruturado em 2012. Resultado: A maioria era do sexo feminino (58\%), $82,2 \%$ solteiros e $40,4 \%$ católicos. Poucos dialogavam com seus pais, sendo $14,4 \%$ conversavam com os pais sobre suas vidas e $11,1 \%$ conversam sobre sexo com os pais. Sobre métodos contraceptivos, $86,7 \%$ conhece algum método contraceptivo, 83,9\% conhecem a camisinha e $33,6 \%$ anticoncepcional oral ou injetável como forma de prevenção. Do total, $45 \%$ deles tinham vida sexual ativa e faziam uso de camisinha masculina. A média de idade de início de relação sexual desses adolescentes foi 12,5 anos de idade e 13,1 anos para os adolescentes que já haviam engravidado alguém. Os amigos são os primeiros a serem procurados pelos adolescentes para contar a notícia da gravidez $(28,5 \%)$, seguidos pela mãe $(2,8 \%)$. Conclusão: Os resultados mostram realidade similar a literatura estudada e apontam para a necessidade de estratégias de educação voltadas para a saúde sexual e reprodutiva dos adolescentes, englobando profissionais de saúde, professores e família, bem

${ }^{1}$ Discente do curso de graduação em Medicina pela Universidade Federal de Alagoas (UFAL). Autora correspondente: Av. Menino Marcelo, 5585. Residencial Parque Maceió. B1. 2, Apto. 1308. Antares. Maceió, Alagoas. Brasil. 57046-000. E-mail: sheyla.acs@gmail.com

${ }^{2}$ Médico. Mestre em Saúde da Criança pela Universidade Federal de Alagoas (UFAL).

${ }^{3}$ Discente do curso de graduação em Medicina pela Universidade Federal de Alagoas (UFAL). 
como o dialogo, para construção de ações que promovam a saúde integral sexual e integral do adolescente

\section{Descritores:}

Sexualidade;

Adolescente; Anticoncepção.

\section{Abstract}

Objective: To describe aspects of the sexual life of adolescent students. Method: Descriptive, cross-sectional, quantitative study with a random sample of 556 students, both genders, from 10 to 18 years old, from three public schools in Maceió/AL. Data were collected through a semi-structured questionnaire in 2013. Results: The majority were female (58\%), $82.2 \%$ were single and $40.4 \%$ were Catholic. Few conversed with their parents: $14.4 \%$ talked to their parents about their lives and $11.1 \%$ talked about sex with them. Regarding contraceptive methods, $\quad 86.7 \%$ knew some contraceptive method, $83.9 \%$ knew condom and $33.6 \%$ knew oral or injectable contraceptives as a form of prevention. Of the total, $45 \%$ of them had an active sex life and used a male condom. The mean age of sexual initiation of these adolescents was 12.5 years old and 13.1 years for adolescents who have already impregnated someone. The friends are the first to be sought by teenagers to tell the news of the pregnancy (28.5\%), followed by the mother (2.8\%). Conclusion: The results show a similar reality to the literature studied and point to the need for education strategies aimed at the sexual and reproductive health of adolescents, including health professionals, teachers and family, as well as the dialogue, to build actions that promote integral sexual health.

\section{Descriptors: Sexuality; Adolescent; Contraception.}

\section{Resumen}

Objetivo: Describir aspectos de la vida sexual de estudiantes adolescentes. Método: Estudio descriptivo, transversal, cuantitativo con una muestra aleatoria de 556 estudiantes, de ambos sexos, de 10 a 18 años a partir de tres escuelas públicas en Maceió-AL. Los datos fueron recogidos a través de un cuestionario con preguntas abiertas e cerradas, en 2013. Resultados: La mayoría eran mujeres (58\%), $82,2 \%$ eran solteras y 40,4\% católicos. Pocos dialogaron con sus padres y madres, $14,4 \%$ habló con sus 
madres acerca de sus vidas y $11,1 \%$ habla de sexo con sus padres. Acerca de la anticoncepción, 86,7\% conoce algún método anticonceptivo, 83,9\% conocían los condones y 33,6\% anticonceptivos orales o inyectables para la prevención. Del total, $45 \%$ de ellos eran sexualmente activos y hacían uso del condón masculino. La edad media de inicio de las relaciones sexuales fue de 12, 5 años y 13,1 años para las adolescentes que se quedaron embarazadas. Los amigos son los primeros buscados por los adolescentes para contar la noticia del embarazo (28,5\%), seguido por la madre (2,8\%). Conclusión: Los resultados muestran la realidad similar a literatura estudiada y apuntan a la necesidad de estrategias de educación dirigidos a la salud sexual y reproductiva de los adolescentes, la participación de profesionales de la salud, maestros y familiares, así como el diálogo para la construcción de acciones que promuevan salud integral sexual e integral de los adolescentes.

Descriptores: Sexualidad; Adolescente; Anticoncepción.

\section{Introdução}

A adolescência é a fase da vida em que o indivíduo se encontra em um processo de grandes aprendizagens, estando mais aberto à adoção de novos comportamentos, o que justifica a priorização desse público prioritário para a educação sexual e educação para a saúde ${ }^{(1)}$.

A sexualidade é um componente intrínseco da pessoa e aspecto fundamental na saúde de adolescentes e jovens. A sexualidade transcende o aspecto meramente biológico, manifestando-se também como um fenômeno psicológico e social, fortemente influenciado pelas crenças e valores pessoais e familiares, normas morais e tabus da sociedade ${ }^{(2)}$.

Para que possa aprender a respeito de sexualidade e criar a seu próprio conceito sobre o tema o jovem precisa receber e assimilar as informações que lhe são passadas; no entanto percebe-se que às vezes as informações sobre sexualidade, transmitidas aos jovens, costumam fundir-se aos aspectos relativos a reprodução humana, abordando apenas aspectos biológicos, em detrimento de outras informações importantes para discussão e entendimento do que é sexualidade. Assim, ensina-se sobre espermatozoides, óvulos, ovários, 
fecundação, gestação e parto e nada ou bem pouco sobre o coito, o orgasmo, a relação sexual, a anatomia do prazer ou ainda os meios de prevenção das doenças $^{(3)}$.

Essa visão simplista e biológica da sexualidade acaba gerando um comportamento de risco nos jovens que iniciam a vida sexual, pois eles não têm conhecimento da importância da relação sexual e de toda a responsabilidade que está atrelada a este ato, sendo a gravidez na adolescência uma das maiores preocupações atuais.

O interesse sobre o comportamento contraceptivo de adolescentes brasileiros tem crescido em decorrência do fato de que, em todas as regiões do País, vem decaindo a taxa de fecundidade, exceto nesta faixa etária. Embora a gravidez na adolescência não seja um fenômeno recente, sua importância como problema de saúde pública vem aumentando, justificando a realização de estudos sobre contracepção nesta etapa da vida ${ }^{(4)}$.

Embora os contraceptivos orais e de barreia estejam disponíveis no sistema de saúde pública do Brasil, há mais atenção para a contracepção medicalizada, faltando uma educação sexual eficaz. Muitas jovens apresentam preocupação com os efeitos colaterais dos contraceptivos orais além de que neste momento, os jovens podem não apresentar maturação psicológica adequada para assumir a responsabilidade por sua saúde ${ }^{(5)}$.

A gravidez na adolescência é um problema de saúde pública tanto no Brasil como em muitos outros países do mundo. Sua importância transcendeu a prática assistencial, dado seu aumento no final do século passado. Para entender os possíveis fatores etiológicos ligados ao incremento das gestações nessa faixa etária, é preciso perceber a complexidade e a multicausalidade desses fatores, que tornam os adolescentes especialmente vulneráveis a essa situação ${ }^{(6)}$.

A maternidade no início da vida reprodutiva antecipa a maturidade biológica, e precipita momentos socialmente institucionalizados para a reprodução, com claras implicações para a constituição de família e a organização social dominante ${ }^{(6)}$. Na adolescência a gravidez é considerada de alto risco, porque pode propiciar o aparecimento de uma série de complicações para mãe e para o feto, pelas alterações biológicas, psicológicas, sociais e culturais que podem $\operatorname{advir}^{(7)}$. 
Uma dessas complicações podem estar associada ao abortamento provocado, que é praticado pelas mulheres, em contexto clandestino, no Brasil, sendo também, considerado uma questão de saúde pública ${ }^{(4,8-11)}$.

Atualmente, uma em cada cinco crianças nasce de mães adolescentes, com idade entre 15 e 19 anos, na América Latina e no Caribe; no Brasil, um em cada cinco nascimentos ocorre com mães com idade entre 10 e 19 $\operatorname{anos}^{(12)}$.

Segundo a W.H.O 16 milhões de adolescentes entre 15 e 19 anos se tornam mães a cada ano, e a gravidez na adolescência é mais comum entre meninas pobres e menos educadas e em países de renda baixa e média. As complicações da gravidez e do parto são a principal causa de morte em mulheres de 15-19 anos em países de baixa renda, onde ocorre cerca de 3 milhões de abortos inseguros anualmente ${ }^{(8)}$.

A gravidez na adolescência reforça o ciclo vicioso da pobreza e da falta de saúde: mães adolescentes, em geral deixam a escola, e são menos propensos do que seus pares para desenvolver habilidades vocacionais devido ao cuidado com o bebê $\hat{e}^{(8)}$.

No Brasil, as ações oriundas da obstetrícia $^{(13)}$ representam o segundo lugar em causas de internações. Gravidez, parto e puerpério representam 9,4\% de internações de jovens entre 10 e 14 anos e $61,4 \%$ entre jovens de 15-19 anos. Em Alagoas esse número sobe para $16 \%$ entre jovens de 10-14 anos e $74,4 \%$ entre jovens de 15-19 anos. Na capital, Maceió, a obstetrícia ocupa o primeiro lugar em número de internações com $29,5 \%$ das causas, sendo que gravidez, parto e puerpério representam $14,2 \%$ das causas entre jovens de 10-14 anos e 78,8\% das causas entre jovens de $15-19$ anos $^{(13)}$.

Diante destes fatores justifica-se este estudo que tem como objetivo descrever aspectos da vida sexual de estudantes adolescentes.

\section{Método}

Trata-se de um estudo descritivo, transversal, quantitativo, realizado nos anos de 2012 e 2013, em três escolas públicas da cidade de Maceió.

A amostra foi aleatória, não probalística, composta por 556 estudantes, de ambos os sexos, entre 10 e 18 anos de idade. As escolas foram selecionadas por conveniência, procurando pesquisar bairros diferentes e da periferia da cidade de Maceió. Anterior à pesquisa foi mantido contato 
com a direção dos colégios envolvidos e após aprovação foi disponibilizado aos estudantes o TCLE para os pais assinarem e no momento da pesquisa foi entregue o termo de assentimento ao jovem para sua concordância.

Usou-se um questionário anteriormente usado em tese de doutorado $^{(14)}$, contendo três sessões: caracterização dos adolescentes, aspectos da vida sexual e sobre abortamento. $\mathrm{O}$ instrumento foi aplicado em horário disponibilizado pela escola, em sala de aula, em momento específico para a pesquisa. Os questionários foram entregues $\mathrm{e}$ guardados em envelope saco, de modo que não houvesse identificação dos respondentes.

Foram exclusos da pesquisa os questionários respondidos sem nenhum dado sobre sexualidade e com letras ilegíveis. Os dados foram analisados no programa Epi Info versão 5.3.3, com IC a $95 \%$. O projeto foi aprovado pelo CEP da UFAL, sob processo de número $1378 / 12$.

\section{Resultados}

Os adolescentes da pesquisa são em sua maioria do sexo feminino
$(58 \%)$, solteiros $(82,2 \%)$, católicos $(40,4 \%)$, não trabalham $(87,9 \%)$ e residem com os pais $(86,7 \%)$. (Tabela $1)$.

Sobre diálogo, apenas 14,4\% dos adolescentes informara conversar sempre ou frequentemente sobre suas vidas com seus pais, este número cai para $11,1 \%$ quando o assunto é sobre sexo. Os meninos (60\%) que engravidaram alguém afirmam que raramente conversam sobre suas vidas com os pais. (Tabela 02).

Quando existe a conversa sobre sexo a mãe é a principal pessoa escolhida. Ao se investigar o diálogo e a ocorrência de uma gravidez, verifica-se que $41,7 \%$ das meninas que já engravidaram, e 33,3\% dos meninos que já engravidaram alguém, conversam com os pais para $41,1 \%$ das meninas que nunca engravidaram e 29,3\% dos meninos que não engravidaram ninguém.

A escolaridade dos pais desses adolescentes é principalmente o ensino fundamental $(48,9 \%$ dos pais e $55 \%$ das mães), poucos possuem o ensino superior $(15,1 \%$ dos pais e $7,7 \%$ das mães). 
Tabela 01. Caracterização dos adolescentes estudados segundo sexo, estado civil, religião, trabalho e residência.

\begin{tabular}{|c|c|c|}
\hline Caracterização & $n=556$ & $\%$ \\
\hline \multicolumn{3}{|l|}{ Sexo } \\
\hline Feminino & 323 & 58,0 \\
\hline Masculino & 210 & 37,7 \\
\hline Não informado & 23 & 4,3 \\
\hline \multicolumn{3}{|l|}{ Estado Civil } \\
\hline Casado & 39 & 7,0 \\
\hline Solteiro & 457 & 82,2 \\
\hline Divorciado & 5 & 0,8 \\
\hline Separado & 1 & 0,2 \\
\hline Não informado & 54 & 9,2 \\
\hline \multicolumn{3}{|l|}{ Religião } \\
\hline Católico & 255 & 40,4 \\
\hline Evangélico & 188 & 33,8 \\
\hline Espírita & 1 & 0,2 \\
\hline Mormo & 2 & 0,4 \\
\hline Candomblé & 1 & 0,2 \\
\hline Outros* & 12 & 2,1 \\
\hline Não informado & 97 & 22,9 \\
\hline \multicolumn{3}{|l|}{ Trabalha } \\
\hline Sim & 67 & 12,1 \\
\hline Não & 489 & 87,9 \\
\hline Não informado & 0 & - \\
\hline \multicolumn{3}{|l|}{ Com quem mora? } \\
\hline Familiares & 14 & 2,5 \\
\hline Pais & 479 & 86,7 \\
\hline Avós & 30 & 5,4 \\
\hline Marido/esposa & 9 & 1,6 \\
\hline Irmãos & 2 & 0,3 \\
\hline Tios & 11 & 2,0 \\
\hline Namorada & 1 & 0,2 \\
\hline Não informado & 7 & 1,8 \\
\hline
\end{tabular}

Da amostra, $86,7 \%$ dos contraceptivos mais conhecidos entre os adolescentes afirmaram conhecer algum adolescentes foram a camisinha $(78,9 \%)$ método contraceptivo, sendo o e o anticoncepcional oral e/ou injetável preservativo feminino ou masculino o $(49,5 \%)$, que são também os mais método contraceptivo mais conhecido utilizados, porém numa proporção bem $(83,9 \%)$, seguido pelo anticoncepcional menor: a camisinha é utilizada por $45 \%$ oral ou injetável $(33,6 \%)$. Os métodos dos adolescentes com vida sexual ativa. 
Tabela 02. Diálogo dos adolescentes com os pais.

\begin{tabular}{lcccccc}
\hline \multicolumn{1}{c}{ DIÁLOGO } & \multicolumn{2}{c}{ Menina } & \multicolumn{2}{c}{ Menino } & Ignorado \\
& $\mathrm{n}$ & $\%$ & $\mathrm{n}$ & $\%$ & $\mathrm{n}$ \\
Conversa sobre a vida com os pais & & & & & \\
$\quad$ Às vezes & 100 & 32,2 & 69 & 35,7 & \\
Frequentemente & 13 & 2,58 & 7 & 3,6 & \\
$\quad$ Raramente & 157 & 52,76 & 100 & 51,9 & \\
$\quad$ Sempre & 51 & 12,46 & 17 & 8,8 & \\
Total & 292 & 100 & 193 & 100 & 69 \\
Conversa sobre sexo com os pais & & & & & \\
Às vezes & 63 & 26,6 & 49 & 29,7 & \\
Frequentemente & 13 & 5,5 & 1 & 0,6 & \\
$\quad$ Raramente & 140 & 59,1 & 106 & 64,3 & \\
$\quad$ Sempre & 21 & 8,8 & 9 & 5,4 & \\
Total & 237 & 100 & 165 & 100 & 152 \\
\hline
\end{tabular}

Outros métodos citados pelos (1\%), não fazer sexo (1\%), coito adolescentes foram: pílula do dia interrompido $(0,5 \%)$, semente de seguinte $(6 \%)$, DIU $(3,8 \%)$, tabelinha maconha $(0,5 \%)$, lavar os genitais com $(1,4 \%), \quad$ diafragma $\quad(1,4 \%)$, água e sabão (0,5\%). (Gráfico 01).

responsabilidade/cuidado $(1,4 \%)$, aborto

\section{Gráfico 01. Métodos contraceptivos citados pelos adolescentes.}

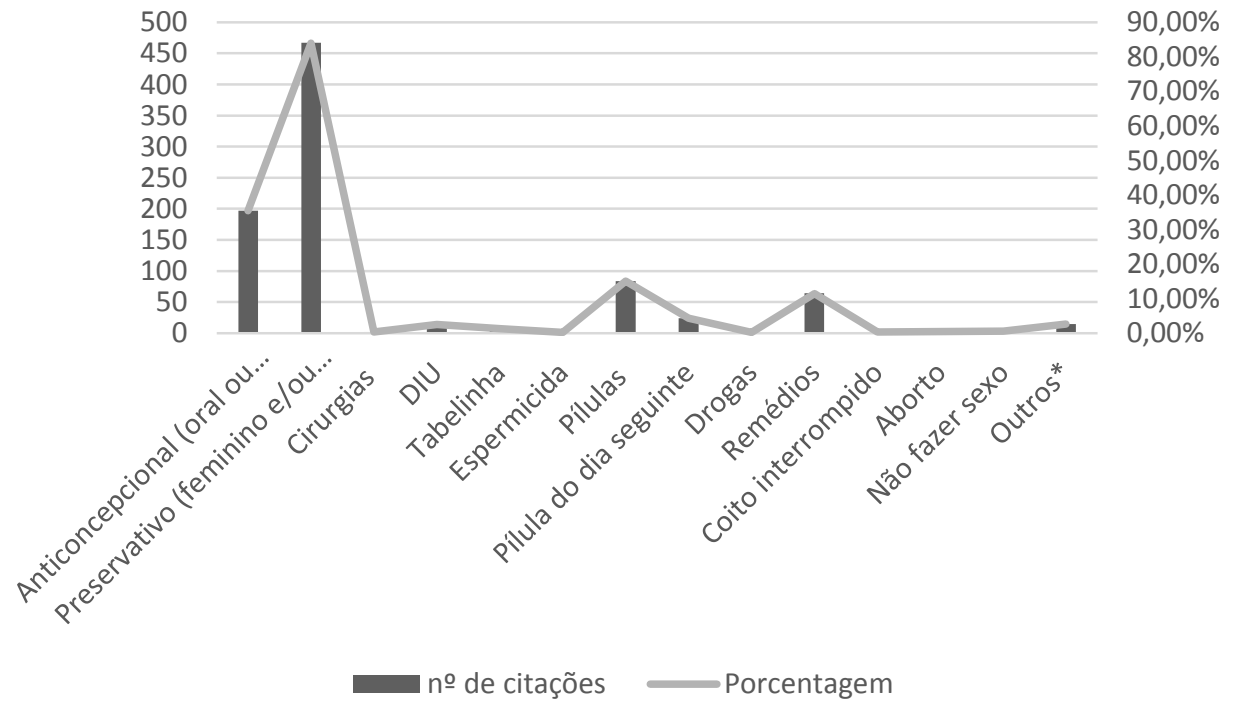


Em relação a sexualidade $38,5 \%$ dos adolescentes afirmaram já ter iniciado a vida sexual, e desses $57 \%$ afirmam utilizar algum método contraceptivo. A média de idade de início de relação sexual desses adolescentes foi 12,5 anos de idade, sendo de 13,1 anos para as adolescentes que já engravidaram ou meninos que já engravidaram alguém. Do total $27 \%$ afirmam conhecer as complicações do aborto. (Tabela 03).

Tabela 03. Aspectos da vida sexual dos adolescentes.

\begin{tabular}{lll}
\hline & \multicolumn{2}{c}{ Total $=214$} \\
Método contraceptivo & $\mathrm{n}$ & $\%$ \\
Usa & 122 & 57 \\
Não usa & 56 & 26 \\
Ignorado & 36 & 17 \\
Gravidez & & \\
Sim & 27 & 13 \\
Não & 170 & 79 \\
Ignorado & 17 & 8 \\
Conhecimento das complicações do aborto & & \\
Sim & 58 & 27 \\
Não & 77 & 36 \\
Não informado & 79 & 37 \\
\hline
\end{tabular}

Entre os jovens que informaram ter engravidado (meninas), ou engravidado alguém (meninos), 80\% conhecem métodos contraceptivos, porém $38,9 \% \quad(36,4 \%$ das meninas e $60 \%$ dos meninos) não usam nenhum método.

Os métodos mais conhecidos são a camisinha masculina e/ou feminina (44\%) e os anticoncepcionais (41\%), que também são os mais utilizados por esses adolescentes, $72 \%$ afirmaram que não planejaram a gravidez e 18,5\% afirmaram já ter ocorrido mais de uma gravidez (Tabela 04).

Todos esses que afirmaram a gravidez, disseram possuir companheiro fixo, $35 \%$ moram juntos, $84,1 \%$ com outras pessoas da família, a maioria $(62 \%)$ reside com os pais de um dos cônjuges. 


\section{Tabela 04. Gravidez e Aborto em Adolescentes do Sexo Feminino}

\begin{tabular}{lcc}
\hline & $\mathrm{n}=21$ & $\%$ \\
Gravidez Planejada & 6 & 28 \\
Sim & 15 & 72 \\
Não & & \\
Recorrência gravidez & 5 & 23 \\
Sim & 16 & 77 \\
Não & & \\
Aborto & 5 & 23 \\
Sim & 5 & 28 \\
Não & 6 & 49 \\
Não informado & 10 & \\
\hline
\end{tabular}

A idade da ocorrência da gravidez entre os meninos foi de no mínimo 12 anos e máximo de 16 anos, sendo a moda de 15 anos; entre as meninas a idade mínima foi 13 anos, a máxima 17 anos e a moda de 13 anos. Os amigos são os primeiros a serem procurados pelos adolescentes para contar a notícia da gravidez $(28,5 \%)$ seguidos pela mãe $(23,8 \%)$. Segundo os dados, $33,3 \%$ das meninas e $14,2 \%$ dos meninos com história de gravidez já fizeram ou pediram para companheira abortar, e 3 adolescentes (14,2\%) afirmam que tiveram complicações por causa do aborto.

\section{Discussão}

O uso da camisinha masculina como método contraceptivo mais usado pelos adolescentes foi um resultado semelhante ao da literatura estudada ${ }^{(14)}$, e pensa-se que esse fato se deve as estratégias públicas de conscientização para o uso desses métodos, atuando como prevenção para a gravidez e para as doenças sexualmente transmissíveis.

$$
\text { Ainda sobre métodos }
$$
contraceptivos, os dados mostram que alguns adolescentes ainda possuem conhecimento errôneo sobre o tipo de métodos anticoncepcionais.

Informações como "lavar a vagina e o pênis com água e sabão" foi uma das respostas encontradas nas perguntas abertas, da pesquisa, o que demonstra deficiência de informação. 
Estudos realizados com adolescentes de 12 a 15 anos evidenciam essa questão, mostrando que são necessárias ações de saúde com escolares a fim de promover o conhecimento da sexualidade entre esses jovens $^{(2)}$.

O aborto, que é crime no Brasil, foi citado como método contraceptivo por $3(0,5 \%)$ adolescentes do nosso estudo (outros do Gráfico 1), confirmando a literatura usada nesse estudo, que apresenta a citação do aborto como método contraceptivo, em conjunto com medicações como o misoprostol, sendo uma maneira ilegal e proibida no Brasil, e assim mesmo, utilizada pelas jovens brasileiras ${ }^{(14)}$.

Tal resultado reforça a necessidade de uma educação sexual mais eficaz e ampla, onde sejam abordados temas como a prevenção da gravidez e as complicações do aborto provocado na adolescência, visto que o aborto é uma das principais causas de morte em adolescentes grávidas em países de baixa renda ${ }^{(1)}$.

Os pais dos adolescentes pesquisados, em sua maioria, possuem apenas o ensino fundamental completo o que pode influenciar no nível de esclarecimento que estes possuam para orientação dos filhos e delineiam um perfil dos escolares como pertencentes a classes econômicas mais baixas.

Segundo alguns estudos, a escolaridade dos pais modifica o conhecimento ou influencia o uso dos métodos anticoncepcionais entre os adolescentes, em alguns casos, apontando para as dificuldades ou mesmo para a ineficiência ou inexistência de diálogos familiares acerca da orientação sexual e, obviamente, para as limitações próprias do núcleo familiar ${ }^{(9)}$.

Segundo os dados, $13 \%$ dos adolescentes com vida sexual ativa afirmaram ter engravidado. Essa é uma média abaixo do valor mundial e um pouco acima da média nacional, que apesar das divergências de valores entre as fontes de consulta giravam em torno de $18 \%$ mundialmente e $10 \%$ nacionalmente.

A idade média de gravidez entre os adolescentes foi de 14,5 anos de idade, que está semelhante aos dados da Organização Mundial de Saúde que aponta uma idade média entre 14 e 17 anos. $\mathrm{Na}$ atualidade, a idade média da primeira relação sexual entre as moças está abaixo dos 15 anos e as adolescentes mais novas, de 10 a 14 anos, em geral, devido à menor idade, ainda têm pensamento abstrato 
incipiente, o que faz com que sejam mais vulneráveis, se expondo a riscos sem prever as consequências ${ }^{(1)}$.

Entres os adolescentes com história de gravidez é possível perceber que apesar da maioria conhecer métodos contraceptivos, uma quantidade considerável $(38,9 \%)$ não usa nenhum tipo de método. Resultado semelhante é encontrado em outros estudos, que relacionam essa falta de prática do uso de métodos contraceptivos com o não poder de compra e o receio na busca pelo serviço de saúde ${ }^{(12)}$.

Apesar de todos os adolescentes com história de gravidez afirmarem que tem companheiro fixo, a maioria mora com os pais ou outros familiares que não os companheiros, o que mostra a dependência dos pais na ocorrência da gravidez. Esse fato pode explicar o medo que os pais dos adolescentes têm de que seus filhos engravidem, pois muitos mudam de companheiro, aumentando o risco de nova gravidez, o que requer uma reorganização financeira da família, considerando a incapacidade desses adolescentes de se sustentarem em razão da pouca idade $^{(5,10)}$.

Estudos mostram que se observa rejeição da família à filha adolescente solteira e grávida, baseada no estigma de mãe solteira. Esses argumentos culturais, e sobre tudo morais, estão assentados nas desigualdades de gênero em relação à repressão sexual e na dupla moral ainda vigente. Existe um maior controle sobre o comportamento sexual feminino do que sobre o masculino, que impõe a responsabilização à mulher pela prevenção da gravidez. Dessa maneira, a culpa é amenizada quando a mulher tem a sorte de o pai da criança assumir a responsabilidade e aceitar a união ${ }^{(11)}$.

O relacionamento sexual, visto como prática inerente ao namoro é geralmente tolerado pelos pais dos adolescentes, mas visto como um costume "moderno" que deveria estar acompanhado dos cuidados com a anticoncepção. Há a concepção de que os homens se aproximam das mulheres com a intencionalidade do relacionamento sexual. A noção de que às mulheres cabia à decisão de ceder ou não aos apelos masculinos estava sedimentada. As adolescentes recebem orientações para não ceder prontamente às investidas masculinas. Os relacionamentos íntimos deveriam ser iniciados somente quando havia a certeza da boa intencionalidade masculina, o que subentendia o 
compromisso da manutenção do vínculo $^{(10)}$.

Verifica-se que é necessário discutir com mais ênfase o tema sexualidade nas escolas, com professores e pessoal capacitado para lidar com o tema, uma vez que, muitos pais não conversam com seus filhos sobre isso. Seja pela falta de tempo ou informação, e muitas vezes pelo pensamento de que poderiam estimular o início da vida sexual. A escola fica então como única fonte que eles têm de buscar conhecimento adequado.

Apesar de todos os adolescentes aqui pesquisados estarem estudando, se constatou que o conhecimento sobre métodos contraceptivos, aborto e suas complicações citadas é oriunda do saber popular e não do conhecimento científico aprendido nas escolas, pois os jovens escreveram em suas respostas afirmações respostas como: "aborto”, “ semente de maconha " e " genéricos" como métodos anticonceptivos e como consequências do aborto " sangramento eterno".

Observou-se que muitos adolescentes possuem conhecimento sobre métodos contraceptivos, gravidez e aborto, no entanto, o assunto ainda é banalizado pelos jovens uma vez que grande parte não utiliza os conhecimentos, perpetuando o ciclo de relação desprotegida, evasão escolar, baixa renda, gravidez indesejada e aumento de doenças sexualmente transmissíveis.

\section{Conclusão}

Torna-se necessário propor e implementar estratégias educacionais voltadas para a saúde sexual e reprodutiva dos adolescentes, englobando profissionais de saúde, professores e família nas escolas. O incentivo do diálogo entre essas esferas possibilitará reflexões acerca da sexualidade, contemplando as perspectivas físicas, psicológicas, emocionais, culturais e sociais, aproximando-se dos adolescentes e alcançando mais pertinência em relação à promoção da saúde integral.

\section{Referências}

1. Teixeira SAM, Taquette SR. Violência e atividade sexual desprotegida em adolescentes menores de 15 anos. Rev. Assoc. Med. Bras. 2010; 56(4): 440-6.

2. Ministério da Saúde (BR). Diretrizes Nacionais para a Atenção Integral a Saúde de Adolescentes e Jovens na Promoção, Proteção e Recuperação da Saúde. Brasília: Ministério da Saúde; 2010: 14-125. 
3. Silva SS, Silva MR, Alves MFP. Sexualidade e Adolescência: é preciso vencer os tabus. Belo Horizonte: Anais do $2^{\circ}$ congresso Brasileiro de extensão Universitária; 2004: 1-9.

4. Freitas KR, Dias SMZ. Percepções de adolescentes sobre sua sexualidade. Texto e Contexto Enferm. 2010; 19(2): 351-7.

5. Bruno ZV, Feitosa FEL, Silveira KP, Morais IQ, Bezerra MF. Reincidência de Gravidez em adolescentes. Revista Bras. Ginecol. Obstet. 2009; 31(10): 480-4.

6. Belo MAV, Silva JLP. Conhecimento, atitude e prática sobre métodos anticoncepcionais entre adolescentes gestantes. São Paulo: Rev de Saúde Pública,2003; 38(4): 479-87.

7. Souza VLC, Corrêa MSM, Souza SL, Beserra MA. Aborto entre adolescentes. Rev Latino Americana de Enferm., 2001; 9(2): 22-47.

8. Kristin M, Sabherwal S. A ReviewofRiskandProtectiveFactors for Adolescent Sexual and Reproductive Health in Developing Countries: An Update. JournalofAdolescent Health 53(2013): 562-572

9. Belo MAV, Silva JLP. Conhecimento, atitude e práticas sobre métodos anticoncepcionais entre adolescentes gestantes. Rev. Saúde Pública, São Paulo. 2004; 38(4): 46987.

10. Hoga LAK, Borges ALV, Alvarez REC. Gravidez na adolescência: valores e reações dos membros da família. Acta Paul Enferm. 2009; 22(6): 779-85.
11. Soares JSF, Lopes MJM. Biografias de gravidez e maternidade na adolescência em assentamentos rurais do Rio Grande do Sul. Rev. Esc. Enferm da USP. 2011; 45(4): 802-10.

12. The Paterniship for Maternal, Newborn\&Chlid Health. PMNCH KnowledgeSummary 22. WHO, 2012: 2-4. Disponível em: http://portal.pmnch.org/Acesso em: 24 de outubro de 2016.

13. Ministério da Saúde (BR). Proporção de internações hospitalares SUS. Indicadores e Dados Básicos. Brasília: Ministério da Saúde; 2009: 296-98.

14. Correia DS, Pontes AP, Calado JC. Egito, EST, Maia EMC. Adolescents: contraceptive knowledge and use, a Brazilian Study. The Scientific World Journal. 2009; 18(9):37-45. 